\title{
Olivenöl schützt vorm Schlaganfall
}

Die Beziehung zwischen Olivenölkonsum und dem Auftreten von Schlaganfällen sollte eine französische Studie aufdecken.

- Wissenschaftler einer 3-Städte-Studie (Bordeaux, Montpellier und Dijon) prüften die Beziehung zwischen Olivenölkonsum (Hauptprobe, $\mathrm{n}=7625$ ) oder Plasma-Ölsäurekonzentration (sekundäre Probe; $\mathrm{n}=1245$ ) und der Schlaganfallinzidenz. Die Probanden waren mindestens 65 Jahre alt und hatten keinen Schlaganfall in der Anamnese.

In der Hauptprobe kam es zu 148 neuen Schlaganfällen. Nach Berücksichtigung aller anderen Faktoren verringerte sich die Wahrscheinlichkeit, einen Schlaganfall zu erleiden um $41 \%$, wenn Olivenöl sowohl zum Kochen und Braten als auch zum Anrichten von Salaten und auf Brot verwendet wurde. In der sekundären Gruppe hatten die mit den höchsten Ölsäurewerten ein um $73 \%$ geringeres Risiko als die mit den niedrigsten Werten.

\section{Kommentar}

Unabhängig von anderen Ernährungsgewohnheiten kann reichlich Olivenöl auf dem Speiseplan das Schlaganfallrisiko älterer Menschen offensichtlich dramatisch senken. Olivenöl wäre eine günstige und einfache Möglichkeit der Schlaganfallprophylaxe.

K. MALBERG =

\footnotetext{
- Samieri, C et al.:

(Equipe Epidémiologie de la Nutrition et des Comportements Alimentaires, INSERM, U897, Université Bordeaux 2, ISPED case 11, 146 rue Léo-Saignat, F-33076 Bordeaux cedex, France; E-mail: Cecilia.Samieri@isped.u-bordeaux2.fr) Olive oil consumption, plasma oleic acid, and stroke incidence.

The Three-City Study. Published before print on June 15, 2011; doi: 10.1212/ WNL.0b013e318220abeb
} 